OPEN ACCESS

Edited by:

Jiufeng Sun,

Guangdong Provincial Center for Disease Control and Prevention, China

Reviewed by:

Salihu Sabiu Musa,

Hong Kong Polytechnic

University, China

Jianhua Hu,

Zhejiang University, China

*Correspondence:

Shengyu Wang

wangshengyu@yeah.net

Tao Jiang

jiangtaotd@163.com

tThese authors have contributed equally to this work and share first

authorship

Specialty section: This article was submitted to Infectious Diseases - Surveillance,

Prevention and Treatment

a section of the journa

Frontiers in Medicine

Received: 11 July 2020

Accepted: 26 April 2021

Published: 28 May 2021

Citation:

Zhang Q, Zhu J, Jia C, Xu S, Jiang T and Wang $S$ (2021) Epidemiology and

Clinical Outcomes of COVID-19 Patients in Northwestern China Who

Had a History of Exposure in Wuhan

City: Departure Time-Originated

Pinpoint Surveillance

Front. Med. 8:582299.

doi: 10.3389/fmed.2021.582299

\section{Epidemiology and Clinical Outcomes} of COVID-19 Patients in Northwestern China Who Had a History of Exposure in Wuhan City: Departure Time-Originated Pinpoint Surveillance

\author{
Qingqing Zhang ${ }^{1 \dagger}$, Jianfei Zhu ${ }^{2,3 t}$, Chenghui Jia ${ }^{2,4 \dagger}$, Shuonan $\mathrm{Xu}^{4 \dagger}$, Tao Jiang ${ }^{2 *}$ and \\ Shengyu Wang ${ }^{1 *}$
}

' Department of Pulmonary and Critical Care Medicine, The First Affiliated Hospital of Xi'an Medical University, Xi'an, China, 2 Department of Thoracic Surgery, Tangdu Hospital, Air Force Military Medical University (Fourth Military Medical University), Xi'an, China, ${ }^{3}$ Department of Thoracic Surgery, Shaanxi Provincial People's Hospital, Xi'an, China, ${ }^{4}$ Department of Cardiothoracic Surgery, The First Affiliated Hospital of Xi'an Medical University, Xi'an, China

Background: Most COVID-19 patients cannot provide a clear exposure time; therefore, this study was designed to predict the progression of COVID-19 by using the definite departure time from Wuhan.

Methods: In this retrospective study, all cases were selected from Northwestern China, which has the lowest population density. As our study endpoints, the incubation period was defined as the date of departure from Wuhan City to the date of symptom onset; we defined the confirmed time as the interval from symptom onset to positive results (samples from the respiratory tract). Both of them were estimated by fitting a Weibull distribution on the departure date and symptom onset. The differences among the variables were analyzed.

Results: A total of 139 patients were ultimately enrolled, and $\sim 10.1 \%$ of patients (14 patients) had no symptoms during their disease course. We estimated the median incubation period to be 4.0 days (interquartile intervals, 2.0-8.0), and the 95th percentile of the distribution was 15.0 days. Moreover, $\sim 5.6 \%$ of patients (7 patients) experienced symptoms 2 weeks after leaving. Furthermore, the estimation median interval from symptom onset to final diagnosis was 4.0 days (interquartile intervals, 2.0-6.0), and the 95th percentile of the distribution was 12.0 days. Finally, the median hospitalization time was 16.0 days, ranging from 3.0 to 45.0 days. Univariate analysis showed that age $(P=0.021)$ and severity status $(P=0.001)$ were correlated significantly with hospitalization time.

Conclusions: We provide evidence that departure time can be used to estimate the incubation and confirmed times of patients infected with COVID-19 when they leave an epidemic area.

Keywords: outcomes, exposure history, departure time, epidemiological terms, COVID-19 


\section{INTRODUCTION}

From December 2019 until March 11, 2020, 3,173 Chinese people died of COVID-19 (1). Compared with the SARS breakout in 2003, COVID-19 presented the following characteristics (2-7): (1) higher infection; (2) lower lethality; (3) infections in the incubation period; (4) asymptomatic patients are also contagious; (5) multiple organ susceptibility. Thus, it is indispensable to investigate the epidemiological characteristics of COVID-19, especially for patients with exposure histories in epidemic areas.

Definite exposure time is critical for analyzing infectious diseases, especially for respiratory tract infectious diseases that were spread through short-range droplets, such as influenza. Previous studies have indicated that the main routes of transmission of COVID-19 were droplets and aerosols (8); other researchers (9) reported that healthy carriers could also transmit the virus. Wuhan city is a megalopolis of high population density in China. Due to the aforementioned factors, we could not obtain definite exposure times, meaning that it was hard to estimate the incubation period of COVID-19. Guan et al. (10), in a retrospective study enrolling 1,099 patients, reported that only 289 patients had information on their specific date of exposure. Other scholars (11) dealt with this situation by choosing the date the first reported patient presented symptoms, which is obviously not rigorous.

Since March 11, the coronavirus has spread to more than 123 countries and regions, with $\sim 132,000$ cases infected with COVID-19, as reported by WHO, spurring WHO to characterize the outbreak as a pandemic. With the global outbreak (12), all countries are facing hard work to prevent and control both domestic epidemics and imported cases from hardest hit areas; however, imported cases or suspicious ones cannot provide definite exposure times, making it hard to calculate the incubation period of patients and to establish the length of time for quarantine and medical observation.

For imported cases, the departure time can be accurately obtained in real-world studies. Therefore, this study was designed to predict the progression of COVID-19 by using definite departure times, which could be easily provided by patients, as the exposure time, and all patients experienced exposure history in Wuhan City. To reduce the possibility of secondary exposure caused by population density and mobility, we selected patients who received treatment at designated hospitals in Northwestern China, the area with the lowest population density.

\section{MATERIALS AND METHODS}

\section{Study Design}

This study was designed to analyze the epidemiological characteristics and clinical outcomes of patients from Northwestern China diagnosed with novel coronavirus pneumonia (NCP) who had history of exposure in Wuhan City. The degree of severity, diagnostic criteria, choice of treatment mode, and discharge standard refer to the 7 th edition of the National New Coronavirus Pneumonia Diagnosis and Treatment Program. To reduce the possibility of secondary exposure caused by population density and mobility, we selected the patients who received treatment at designated hospitals distributed in Northwestern China, the area with the lowest population density. This area includes four autonomous regions and three provinces. All cases enrolled in this study fulfilled the following criteria: (1) had exposure history in Wuhan City; (2) without a definite exposure date; (3) COVID-19 virus nucleic acid results were positive; (4) no direct contact with confirmed or suspected patients after leaving Wuhan City; (5) symptom appearance after leaving Wuhan city; (6) treated at a designated hospital; (7) with definite disease outcome (death or discharge). The last follow-up time was March 11, 2020. This study was approved by the ethics committee of the First Affiliated Hospital of Xi'an Medical University (No. XYYFY2020LSK-026). Written informed consent was waived due to the nature of open-access data, and it was approved by the First Affiliated Human Research Ethics Committee of Xi'an Medical University. All procedures followed were in accordance with the Declaration of Helsinki.

\section{Setting}

These areas are located in Northwestern China, far from Wuhan City. They include four autonomous regions (Inner Mongolia Autonomous Region, Tibet Autonomous Region, Xinjiang Uygur Autonomous Region, and Ningxia Hui Autonomous Region) and three provinces (Gansu Province, Qinghai Province, and Shaanxi Province) (Table 1). These regions and provinces account for $57.5 \%$ of the total territory of China; however, the population density of the area is only 23.8 persons $/ \mathrm{km}^{2}$, which is lower than the national average population density (145.4 persons $/ \mathrm{km}^{2}$ ).

\section{Data Collection}

We obtained the data from the news and press releases reported by the provincial and local municipal Center for Disease Control and Prevention (CDC) or the Health Commission. The date of leaving Wuhan City, the date of symptom onset, the date of diagnosis, the date of discharge, age, gender, and other patientrelated data were extracted from the news and press releases. Four reviewers (JZ, CJ, QZ, and SX) collected the data independently, and data were verified with the National Health Commission and Chinese CDC. Major disagreements between these four doctors were checked by all doctors together.

\section{Definition}

The residents were confirmed to have stayed in Wuhan City more than 2 weeks during the outbreak. The incubation period was defined as the date of departure from Wuhan City to the date of symptom onset or the date of final diagnostic time (asymptomatic patient); we defined the confirmed time as the interval from symptom onset to positive results (samples from the respiratory tract). Hospitalization time is recognized as from final diagnosis to date of discharge or death (Figure 1).

\section{Statistical Analysis}

Categorical variables are summarized as numbers and percentages. We estimated the incubation period and the confirmed time by fitting a Weibull distribution on the dates of departure and symptom onset. The relationship between 
TABLE 1 | Association between clinical characteristics and severity status of patients diagnosed with COVID-19.

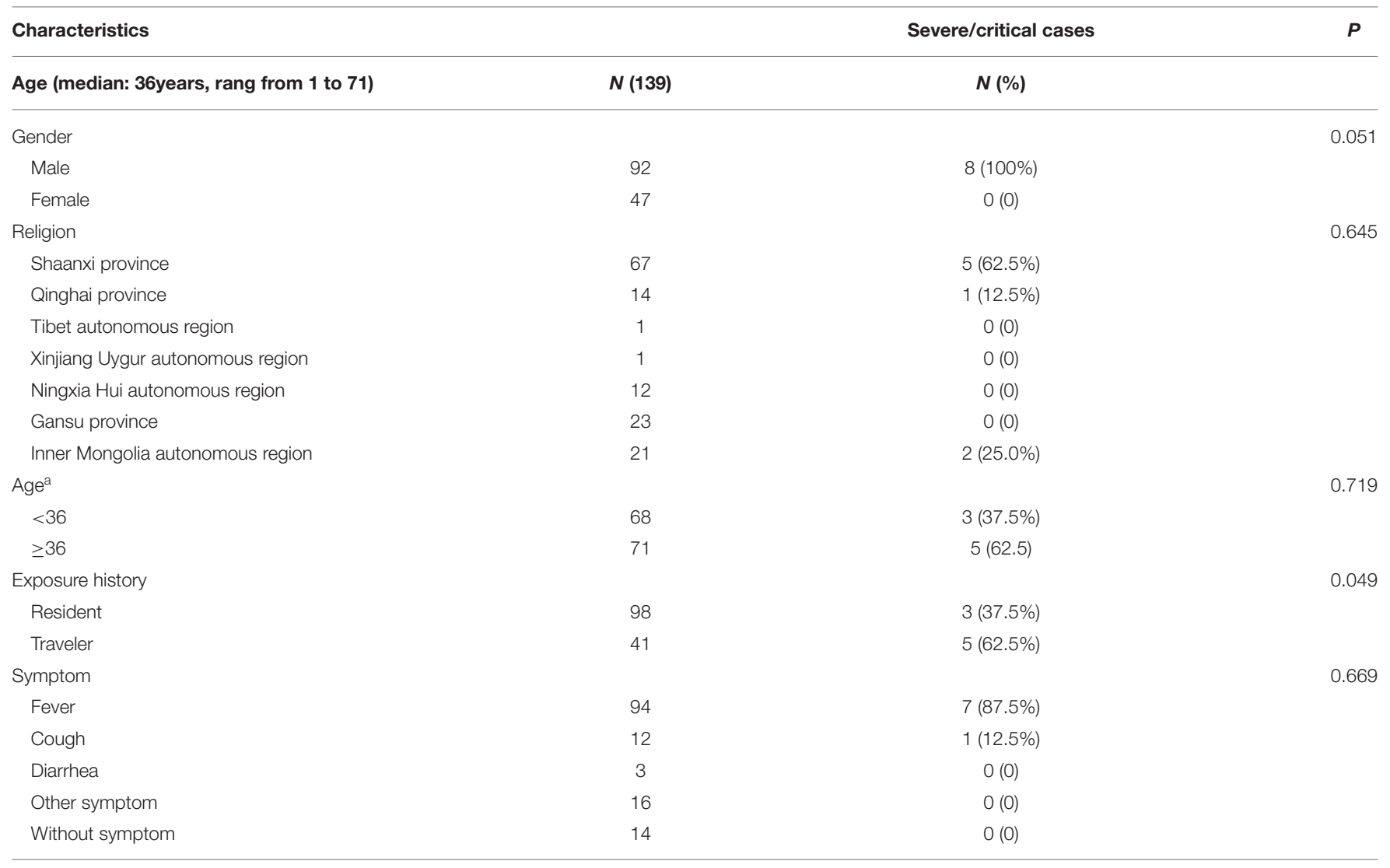

${ }^{a}$ Age: median age.

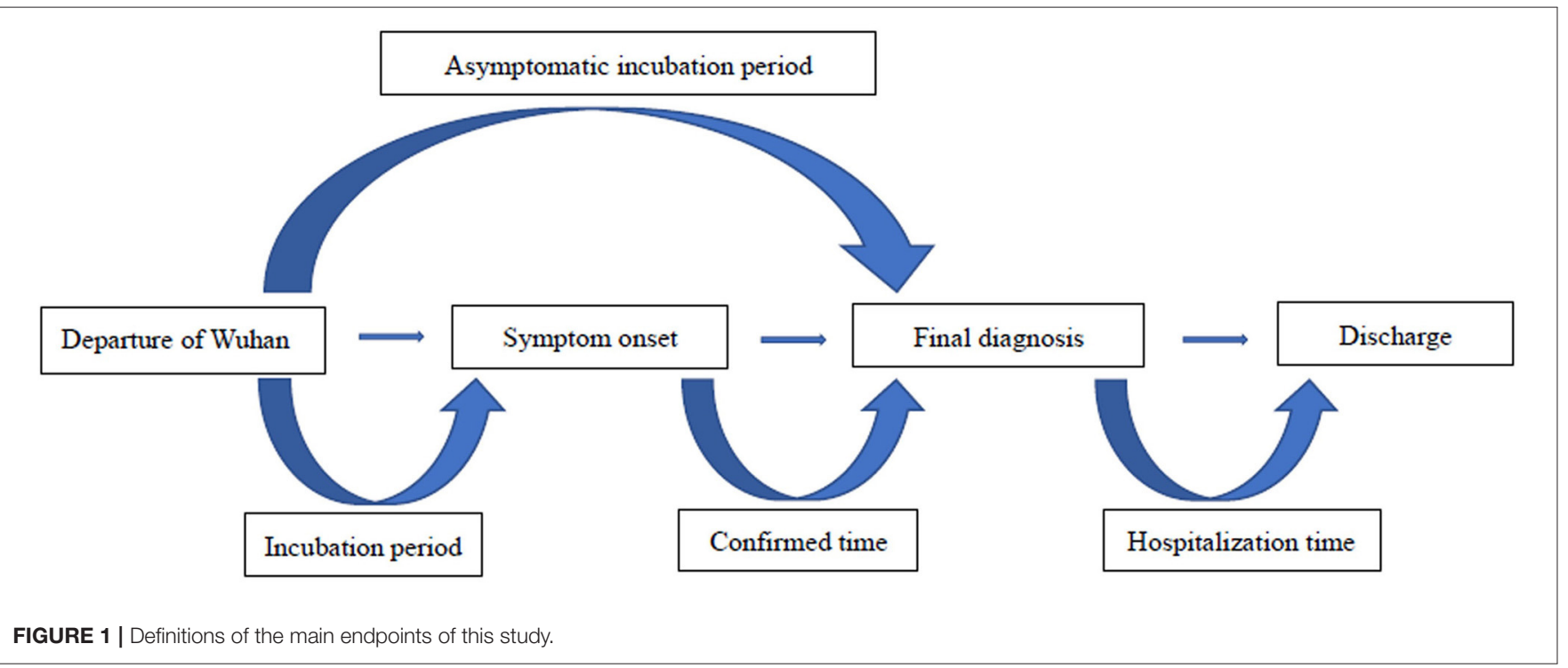

severity status of COVID-19 and clinical characters was analyzed by using a $\chi^{2}$-test. Normal distribution and homogeneity of variances were tested, $T$-test and variance analysis were performed to compare the difference among the variables, and the Mann-Whitney U-test and Kruskal-Wallis H-test were applied when the cases did not fit the normal data distribution. Bilateral $P \leq 0.05$ was considered statistically significant. All analyses were performed using SPSS software 
(version 22.0), and Weibull fitting distribution was estimated by MATLAB 18.0.

\section{RESULTS}

\section{Clinical Characteristics and Severity Status}

As of March 11, 2020, a total of 139 patients diagnosed with COVID-19 were enrolled in this study. All patients were from Northwestern China and were verified to have an exposure history in Wuhan City. Figure 2 shows the time distribution of all patients; the earliest and latest times to leave Wuhan City were January 6, 2020 and January 23, 2020, respectively. Only one patient was provided by both Tibet Autonomous Region and Xinjiang Uygur Autonomous Region. The largest number of patients (67 patients) was provided by Shaanxi Province, accounting for $48.2 \%$ (Table 1 ).

Of the 139 patients, 92 patients were male and 47 patients were female; the median age of the patients was 36 years (range: 1-77 years). Approximately $70.5 \%$ of patients (98 patients) were residents, and 41 patients were travelers. A total of 94 patients had the common symptom of fever $(75.2 \%), \sim 2.4 \%$ of patients (3 patients) presented diarrhea, 12 patients presented only cough $(9.6 \%)$, and $12.8 \%$ of patients presented other symptoms. According to severity status, $\sim 94.2 \%$ of patients were categorized as general, 4 patients $(2.9 \%)$ as severe, and 4 patients $(2.9 \%)$ as critical; severe and critical patients were

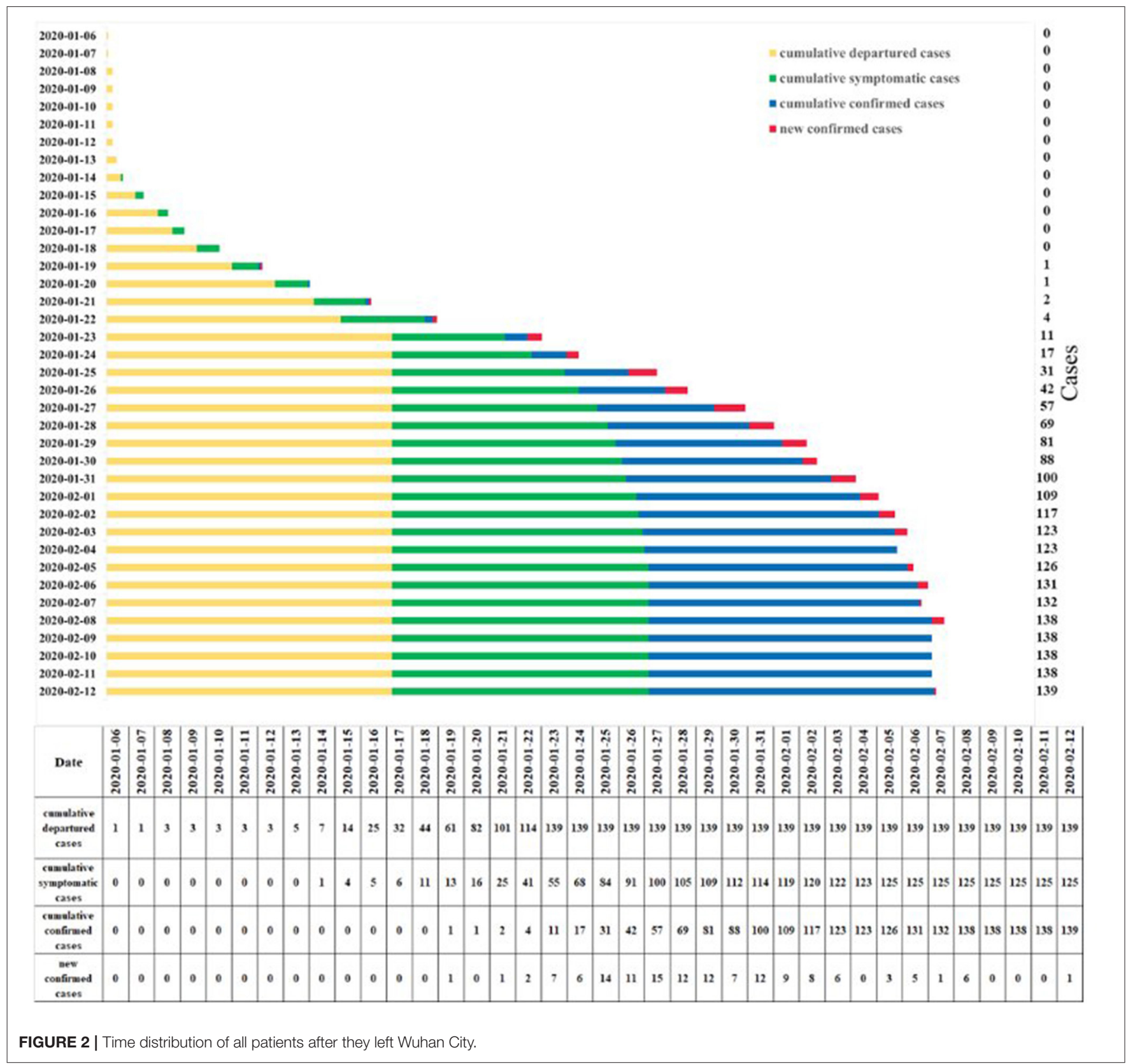


analyzed together. Patients with short-term exposure (travelers) were more likely to develop severe or critical status than those with long-term exposure (residents) (62.5 vs. $37.5 \%, P=0.049$ ). Of the eight severe or critical patients, all of them were male $(P=0.051)$ (Table 1).

\section{Epidemiological Characteristics}

Interestingly, $\sim 10.1 \%$ of patients (14 patients) were healthy carriers, without any symptoms during their disease course. For asymptomatic patients, $\sim 57.1 \%$ of patients (8/14) were determined to be positive for nucleic acid of COVID-19 virus within 5.0 days of leaving Wuhan City. For symptomatic patients, two patients presented onset of symptoms after they were confirmed (1.6\%), and one of them presented symptoms after 9 days. Most patients (75.2\%) had onset of symptoms within a week (94/125), nearly 5.6\% of patients (7 patients) experienced symptoms 2 weeks after leaving, and one patient developed symptoms after 23.0 days. In addition, the peak time of symptom onset emerging after they left the epicenter was on the first day (23 patients) (Figure 3A). All 139 patients diagnosed with COVID-19 were estimated by fitting the Weibull distribution; the median incubation period was 4.0 days (interquartile range, 2.0-8.0), and the 95th percentile of the distribution was 15.0 days (Figure 3B).
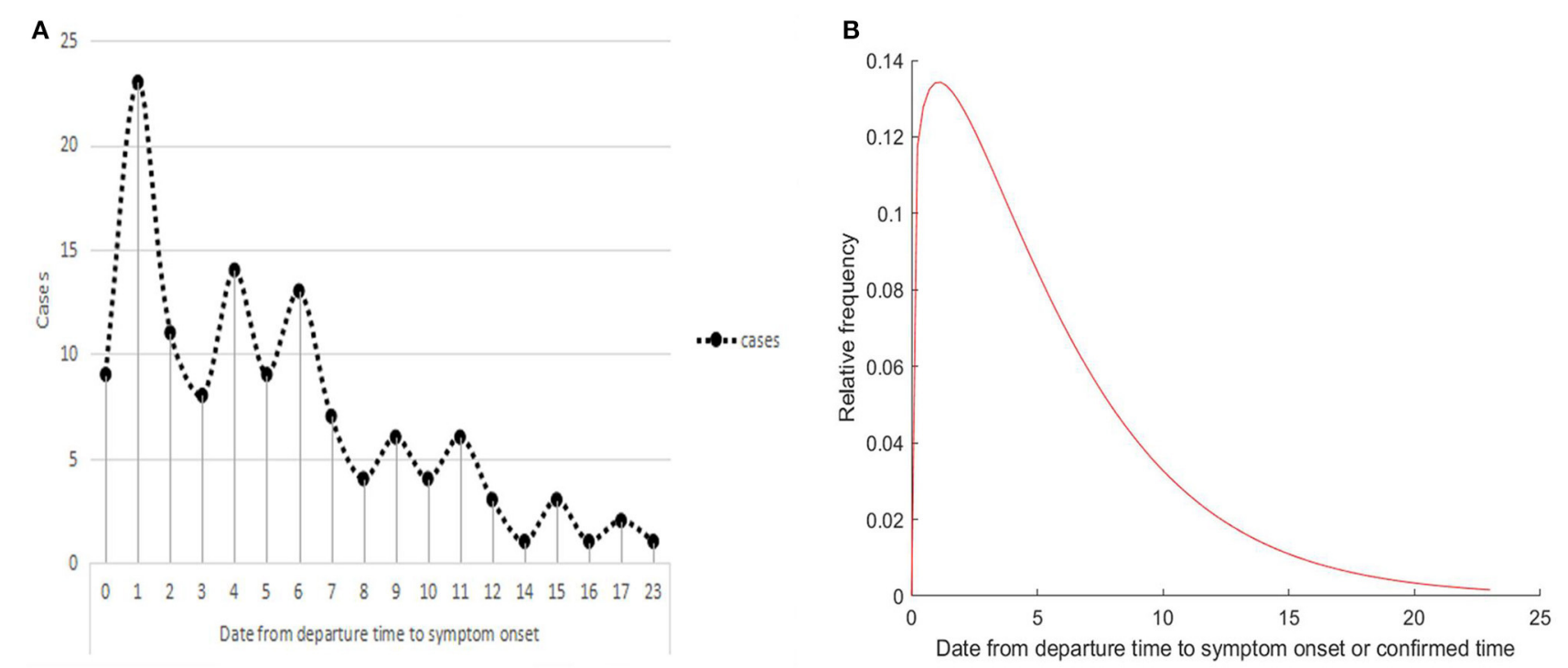

FIGURE 3 | The incubation period of COVID-19. (A) Frequency distribution of incubation periods. (B) Estimation of incubation period by fitting a Weibull distribution.
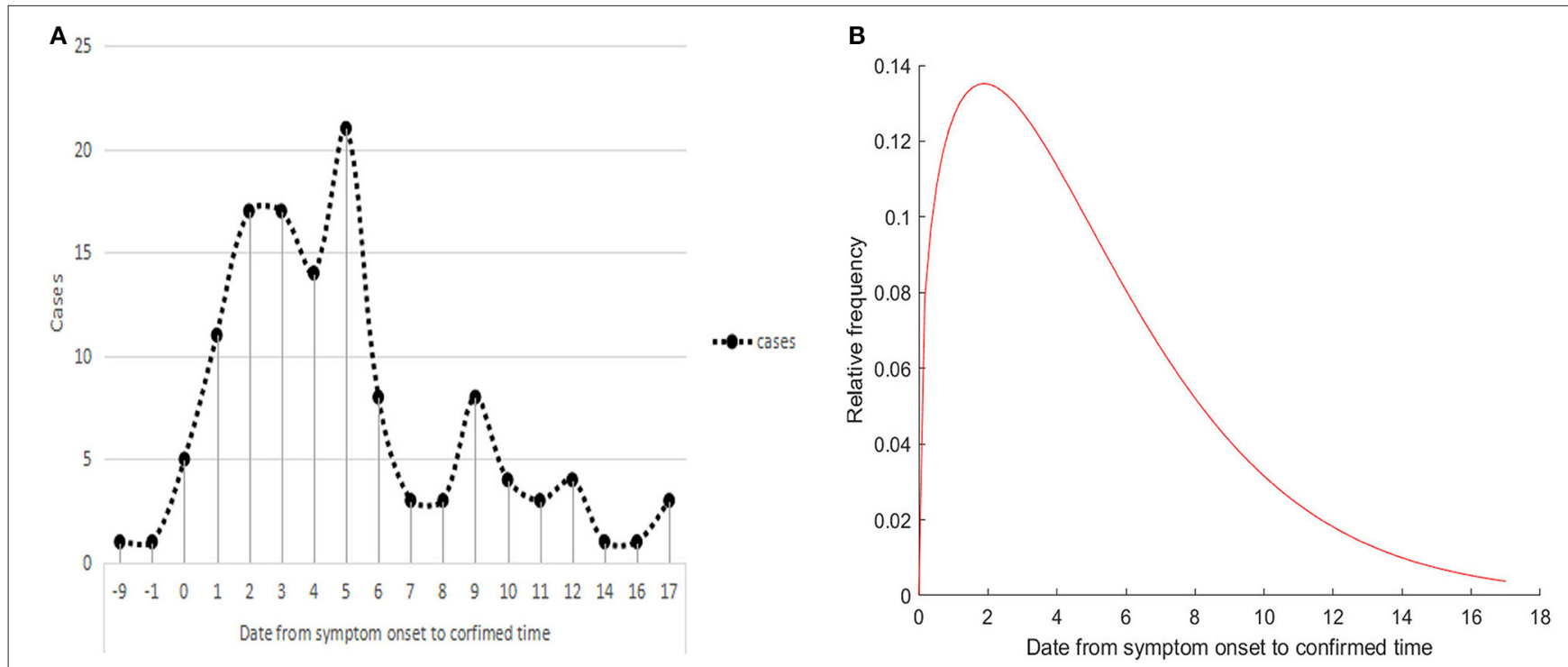

FIGURE 4 | The confirmed time of COVID-19. (A) Frequency distribution of confirmed times. (B) Estimation of the confirmed time by fitting a Weibull distribution. 
Our study purpose was to address the issue of the optimal detection opportunity after symptom onset. The median confirmed time was 4.0 days ( -9 to 17.0 days). Figure $4 \mathrm{~A}$ shows that the peak of confirmed time was on the 5 th day after symptom onset, and $\sim 68.0 \%$ of patients presented positive results within 5 days of symptom onset. For 125 symptomatic patients, the median interval from symptom onset to final diagnosis was 4.0 days (interquartile range, 2.0-6.0), and the 95th percentile of the distribution was 12.0 days (Figure 4B).

The time of positive detection of older patients ( $\geq 36$ years) was later than that of younger patients ( $<36$ years) after symptom onset (5.0 vs. 4.0 days, $P=0.028$ ); in addition, male patients had later positive results detected (5.0 days, interquartile range: $3.0-$ 7.0) compared with female patients (3.0 days, interquartile range: 2.0-5.0, $P=0.051$ ).

\section{Clinical Outcomes}

Since March 11, 2020, all patients have been discharged; they all recovered. The median hospitalization time was 17.0 days, ranging from 3.0 to 45.0 days. Further analyses showed that the following variables were correlated significantly with hospitalization time: age $(P=0.021)$ and severity status $(P=0.001)$ (Table 2).

\section{DISCUSSION}

This retrospective study estimated the incubation period for COVID-19 by using the departure time from Wuhan City as the exposure time because in real-world studies, it is difficult to determine the definite exposure time. We collected the patients from a lower-population-density area in Northwestern China to reduce the possibility of secondary exposure; thus, our data had practical guidance value for preventing and controlling COVID-19, especially for patients or persons with suspicion of infection from the epicenter. Our data showed that the median incubation period of COVID-19 was 4.0 days after leaving the epidemic area.

Our results were basically consistent with other studies (2, 11, 13), although the definition of exposure time was different. A retrospective study from China showed that the mean incubation period of COVID-19 was 4.0 days (95\% CI, 2.07.0); however, while a total of 1,099 patients were enrolled in this study, only 291 had a clear exposure time. All patients were Chinese. Lauer et al. (11) estimated the incubation period of COVID-19 in his study: 181 patients from 24 countries or regions were analyzed, and the median incubation period was estimated to be 5.1 days (95\% CI, 4.5-5.8 days). Other related research reported longer incubation periods than ours. An epidemiological surveillance of early confirmed COVID19 patients in Shanghai (14) showed that the mean incubation period was 6.4 days (95\% CI 5.3-7.6), and the 95th percentile was 13.1 days. Data from Henan Province (15) of China estimated that the average latency of 483 patients was 7.4 days, and over $92.0 \%$ of patients had an incubation period of $<2$ weeks. We also found that $\sim 75.2 \%$ patients developed symptoms within 1 week after leaving the epidemic area (Wuhan City). It was noteworthy that $5.6 \%$ of patients presented symptoms 2 weeks later, while one patient's symptoms appeared 23.0 days later.

Interestingly, $\sim 10.1 \%$ of (14 patients) did not present any clinical symptoms in their disease course. It is controversial whether asymptomatic patients are contagious. A study from

TABLE 2 | The epidemiological characteristics and clinical outcomes of patients diagnosed with COVID-19.

\begin{tabular}{|c|c|c|c|c|c|c|}
\hline Variable & Symptom onset to confirmed time & $\mid \mathbf{Q}^{\mathrm{b}}$ & $P$ & Hospitalization time & $\mid Q^{b}$ & $\boldsymbol{P}$ \\
\hline \multicolumn{7}{|l|}{ In total } \\
\hline Gender & & & 0.051 & & & 0.085 \\
\hline Female & 3 & $(2,5)$ & & 18 & $(13,21)$ & \\
\hline Age $^{\mathrm{a}}$ & & & 0.028 & & & 0.021 \\
\hline$<36$ & 4 & $(2,5)$ & & 15.5 & $(12,19)$ & \\
\hline Exposure history & & & 0.145 & & & 0.881 \\
\hline Resident & 4 & $(2,6)$ & & 16 & $(12,21)$ & \\
\hline Traveler & 5 & $(3,9)$ & & 18 & $(11.5,20)$ & \\
\hline Symptom & & & 0.801 & & & 0.127 \\
\hline Fever & 4 & $(2,6)$ & & 17 & $(13,21)$ & \\
\hline Cough & 4 & $(2,5)$ & & 20 & $(10.25,27.75)$ & \\
\hline General & 4 & $(2,6)$ & & 16 & $(12,20)$ & \\
\hline Severe & 8 & $(1.5,10.75)$ & & 23.5 & $(19.75,31)$ & \\
\hline Critical & 2.5 & $(-0.25,9)$ & & 34 & $(22.25,44.25)$ & \\
\hline
\end{tabular}

${ }^{a}$ Age: median age.

bIQI: interquartile intervals. 
Chinese researchers showed (16) that at least $59.0 \%$ of infection cases in Wuhan City might had not been identified, which may include those who are asymptomatic or who have mild symptoms. The MedRxiv platform published research from American scholars (17) that suggested that there may be a small percentage of infected individuals who are asymptomatic and can transmit the COVID-19 virus. An asymptomatic carrier from Henan Province of China transmitted COVID-19 to her five family members; her incubation period was as high as 19.0 days (9). Moreover, some research has indicated that there was no difference in the virus load between asymptomatic patients and symptomatic patients $(18,19)$. However, other studies showed the opposite result (20). In our study, the results of COVID-19 nucleic acid testing were positive, which indirectly proved that asymptomatic patients are contagious compared with symptomatic patients; thus, we should pay more attention to asymptomatic patients.

Further studies have shown that most asymptomatic patients are categorized as general $(5,21)$, meaning that these patients have better outcomes. However, the next generation of patients who become infected by these asymptomatic individuals might have worse outcomes (22); the specific mechanism of viral pathogenesis is unknown. In our study, for asymptomatic patients, $\sim 68.0 \%$ patients were detected as positive for COVID19 viral nucleic acid within 5.0 days of leaving Wuhan City due to the early detection of suspicious populations by the government. Shao and Shan (23) constructed a SEIR model and suggested that medical examinations should be performed on exposed or potentially exposed individuals.

In concert with recent studies, fever was the most common symptom (75.2\%) diagnosed in this study, which was consistent with the results of the meta-analysis by Sun et al. (24). For patients diagnosed with COVID-19, laboratory test results that did not match clinical symptoms were found in these studies (25-27). Two patients experienced symptoms after they were diagnosed (1.6\%), and one presented symptoms after 9 days; thus, all suspicious populations should be observed dynamically.

This study also focused on the optimal time to detect COVID19 nucleic acids. We found that the median confirmation time was 4.0 days. A recent retrospective study from Tongji Hospital (28) proved that the median time from symptom onset to confirmation was 16.0 days, longer than our data. They also found that $\sim 30.0 \%$ of these patients had positive results for the third time; meanwhile, they found that positive results were detected later in older patients ( $\geq 65$ years) (18.0 vs. 14.0 days, $P<0.001)$, consistent with our data. Our results also indicated that most positive results appeared on the fifth day after symptom onset. Similar to our results, the clinical sensitivity of RT-PCR on swabs taken on the first day to the fifth day after symptom onset was $100 \%$ (29).

We also analyzed the factors that affect the patient's hospitalization time. In our study, the median hospitalization time was 17.0 days, ranging from 3.0 to 45.0 days. Further analyses showed that the following variables were correlated significantly with hospitalization time: age $(P=0.021)$ and severity status $(P=0.001)$, suggesting that age and severity status might been the prognostic factors for patients with COVID-19. A study from China had confirmed that older age associated with patient's in-hospital death (OR: 1.10, 95\% CI: 1.03-1.17, $P=0.0043$ ) (30). The possible explanation was that age and the severity of pneumonia will increase the occurrence of cardiac events after pneumonia, leading to a poor prognosis for patients (31). Therefore, we should pay more attention to these patients.

The limitations of this study should be noted. First, the laboratory results had not been analyzed because data sources from news and press releases were reported by Provincial and Autonomous CDCs. Second, because this was a retrospective study, further analysis was limited due to the small sample size of this study. Finally, although our results were broadly consistent with the related research, patients with exact exposure times should be included in future analyses for comparison.

In conclusion, we provide evidence that departure time can be used to estimate the incubation and confirmed times of patients infected with COVID-19 when they leave an epidemic area. The median of the incubation period was 4 days, and $5.6 \%$ of patients experienced symptoms 2 weeks after leaving. The longest time was 23.0 days from the date of departure, suggesting that the length of time for quarantine and medical observation, now recognized as 2 weeks, might not be sufficient. Moreover, most patients were detected to be positive for viral nucleic acid within 5.0 days of when symptoms appeared. Finally, healthy carriers should be given more attention.

\section{DATA AVAILABILITY STATEMENT}

The original contributions presented in the study are included in the article/supplementary material, further inquiries can be directed to the corresponding author/s.

\section{ETHICS STATEMENT}

The studies involving human participants were reviewed and approved by the ethics committee of the First Affiliated Hospital of Xi'an Medical University (No. XYYFY2020LSK-026). The patients/participants provided their written informed consent to participate in this study.

\section{AUTHOR CONTRIBUTIONS}

TJ and SW participated in study design and study conception. QZ, JZ, and CJ performed data analysis and drafted the manuscript. QZ, JZ, CJ, and SX recruited patients. All authors provided critical review of the manuscript and approved the final draft for publication.

\section{FUNDING}

This work was supported in part by grant from the Shaanxi Province Key Program Fund (2017SF-256). 


\section{REFERENCES}

1. Daily Report of New Coronavirus Pneumonia. Available online at: http://k.sina. com.cn/article_6880107524_19a161c0401900lhp4.html?from=edu (accessed June 30, 2020).

2. Li Q, Guan X, Wu P, Wang X, Zhou L, Tong Y, et al. Early transmission dynamics in Wuhan, China, of novel coronavirus-infected pneumonia. N Engl J Med. (2020) 382:1199-207. doi: 10.1056/NEJMoa20 01316

3. Hui DS, Chan PK. Severe acute respiratory syndrome and coronavirus. Infect Dis Clin North Am. (2010) 24:619-38. doi: 10.1016/j.idc.2010.04.009

4. Huang C, Wang Y, Li X, Ren L, Zhao J, Hu Y, et al. Clinical features of patients infected with 2019 novel coronavirus in Wuhan, China. Lancet. (2020) 395:497-506. doi: 10.1016/S0140-6736(20)30183-5

5. Nishiura H, Kobayashi T, Suzuki A, Jung SM, Hayashi K, Kinoshita R, et al. Estimation of the asymptomatic ratio of novel coronavirus infections (COVID-19). Int J Infect Dis. (2020) 94:154-5. doi: 10.1016/j.ijid.2020. 03.020

6. Wang $\mathrm{S}$, Zhou $\mathrm{X}$, Zhang $\mathrm{T}$, Wang $\mathrm{Z}$. The need for urogenital tract monitoring in COVID-19. Nat Rev Urol. (2020) 17:314-5. doi: 10.1038/s41585-020-0319-7

7. Zubair AS, McAlpine LS, Gardin T, Farhadian S, Kuruvilla DE, Spudich S. Neuropathogenesis and neurologic manifestations of the coronaviruses in the age of coronavirus disease 2019: a review. JAMA Neurol. (2020) 77:1018-27. doi: 10.1001/jamaneurol.2020.2065

8. Liu Y, Ning Z, Chen Y, Guo M, Liu Y, Gali NK, et al. Aerodynamic analysis of SARS-CoV-2 in two Wuhan hospitals. Nature. (2020) 582:557-60. doi: 10.1038/s41586-020-2271-3

9. Bai Y, Yao L, Wei T, Tian F, Jin D Y, Chen L, et al. Presumed asymptomatic carrier transmission of COVID-19. JAMA. (2020) 323:1406-7. doi: 10.1001/jama.2020.2565

10. Guan WJ, Ni Z Y, Hu Y, Liang W H, Ou C Q, He J X, et al. Clinical characteristics of coronavirus disease 2019 in China. N Engl J Med. (2020) 382:1708-20. doi: 10.1056/NEJMoa2002032

11. Lauer SA, Grantz K H, Bi Q, Jones F K, Zheng Q, Meredith HR, et al. The incubation period of coronavirus disease 2019 (COVID-19) from publicly reported confirmed cases: estimation and application. Ann Intern Med. (2020) 172:577-82. doi: 10.7326/M20-0504

12. Coronavirus Latest: WHO Describes Outbreak as Pandemic. Available online at: https://www.nature.com/articles/d41586-020-00154-w (accessed March 11, 2020).

13. Ki M, Task Force for-nCoV. Epidemiologic characteristics of early cases with 2019 novel coronavirus (2019-nCoV) disease in Korea. Epidemiol Health. (2020) 42:e2020007. doi: 10.4178/epih.e2020007

14. Lu HZ, Ai JW, Shen YZ, Li Y, Li T, Zhou X, et al. A descriptive study of the impact of diseases control and prevention on the epidemics dynamics and clinical features of SARS-CoV-2 outbreak in Shanghai, lessons learned for metropolis epidemics prevention. medRxiv [Preprint]. (2020). doi: 10.1101/2020.02.19.20025031

15. Wang P, Lu JA, Jin Y, Zhu M, Wang L, and Chen S. Statistical and network analysis of 1212 COVID-19 patients in Henan, China. Int J Infect Dis. (2020) 95:391-98. doi: 10.1016/j.ijid.2020.04.051

16. Wang CL, Liu L, Hao XJ, Guo H, Wang Q, Huang J, et al. Evolving epidemiology and impact of non-pharmaceutical interventions on the outbreak of coronavirus disease 2019 in Wuhan, China. medRxiv [Preprint]. (2020). doi: 10.1101/2020.03.03.20030593

17. Sanche S, Lin YT, Xu CG, Romero-Severson E, Hengartner N, Ke R. The novel coronavirus, 2019-nCoV, is highly contagious and more infectious than initially estimated. medRxiv [Preprint]. (2020). doi: $10.1101 / 2020.02 .07 .20021154$
18. Zou L, Ruan F, Huang M, Liang L, Huang H, Hong Z, et al. SARS-CoV-2 viral load in upper respiratory specimens of infected patients. N Engl J Med. (2020) 382:1177-9. doi: 10.1056/NEJMc2001737

19. Rothe C, Schunk M, Sothmann P, Bretzel G, Froeschl G, Wallrauch C, et al. Transmission of 2019-nCoV infection from an asymptomatic contact in Germany. N Engl J Med. (2020) 382:970-1. doi: 10.1056/NEJMc2001468

20. Anderson RM, Heesterbeek H, Klinkenberg D, Hollingsworth TD. How will country-based mitigation measures influence the course of the COVID-19 epidemic? Lancet. (2020) 395:931-4. doi: 10.1016/S0140-6736(20)30567-5

21. Wang Y, Liu Y, Liu L, Wang X, Luo N, Ling L. Clinical outcome of 55 asymptomatic cases at the time of hospital admission infected with SARS-Coronavirus-2 in Shenzhen, China. J Infect Dis. (2020) 221:1770-4. doi: 10.1093/infdis/jiaa119

22. Hu ZL, Song C, Xu CJ, Jin GF, Chen YL, Xu X, et al. Clinical characteristics of 24 asymptomatic infections with COVID-19 screened among close contacts in Nanjing, China. Sci China Life Sci. (2020) 63:70611. doi: $10.1007 /$ s11427-020-1661-4

23. Shao P, Shan YJ. Beware of asymptomatic transmission: study on 2019-nCoV prevention and control measures based on extended SEIR model. bioRxiv [Preprint]. (2020). doi: 10.1101/2020.01.28.923169

24. Sun P, Qie S, Liu Z, Ren J, Li K, Xi J. Clinical characteristics of hospitalized patients with SARS-CoV-2 infection: a single arm meta-analysis. J Med Virol. (2020) 92:612-7. doi: 10.1002/jmv.25735

25. Chan JF, Yuan S, Kok KH, To KK, Chu H, Yang J, et al. A familial cluster of pneumonia associated with the 2019 novel coronavirus indicating person-toperson transmission: a study of a family cluster. Lancet. (2020) 395:514-23. doi: 10.1016/S0140-6736(20)30154-9

26. Pan XF, Chen DX, Xia Y, Wu XW, Li TS, Ou XT, et al. Asymptomatic cases in a family cluster with SARS-CoV-2 infection. Lancet Infect Dis. (2020) 20:410-1. doi: 10.1016/S1473-3099(20)30114-6

27. Ling Z, Xu X, Gan Q, Zhang L, Luo L, Tang X, et al. Asymptomatic SARS-CoV2 infected patients with persistent negative CT findings. Eur J Radiol. (2020) 126:108956. doi: 10.1016/j.ejrad.2020.108956

28. Xiao AT, Tong YX, Gao C, Zhu L, Zhang YJ, Zhang S, et al. Dynamic profile of RT-PCR findings from 301 COVID-19 patients in Wuhan, China: a descriptive study. J Clin Virol. (2020) 127:104346. doi: 10.1016/j.jcv.2020.104346

29. Woelfel R, Corman VM, Guggemos W, Seilmaier M, Zange S, Mueller MA, et al. Clinical presentation and virological assessment of hospitalized cases of coronavirus disease 2019 in a travel-associated transmission cluster. medRxiv [Preprint]. (2020). doi: 10.1101/2020.03.05.20030502

30. Zhou F, Yu T, Du R, Fan G, Liu Y, Liu Z, et al. Clinical course and risk factors for mortality of adult inpatients with COVID-19 in Wuhan, China: a retrospective cohort study. Lancet. (2020) 395:1054-62. doi: 10.1016/S0140-6736(20)30566-3

31. Corrales-Medina VF, Musher DM, Shachkina S Chirinos JA. Acute pneumonia and the cardiovascular system. Lancet. (2013) 381:496-505. doi: 10.1016/S0140-6736(12)61266-5

Conflict of Interest: The authors declare that the research was conducted in the absence of any commercial or financial relationships that could be construed as a potential conflict of interest.

Copyright (c) 2021 Zhang, Zhu, Jia, Xu, Jiang and Wang. This is an open-access article distributed under the terms of the Creative Commons Attribution License (CC $B Y)$. The use, distribution or reproduction in other forums is permitted, provided the original author(s) and the copyright owner(s) are credited and that the original publication in this journal is cited, in accordance with accepted academic practice. No use, distribution or reproduction is permitted which does not comply with these terms. 